Working

Paper

Series

Optimal Pricing and Quality of Academic Journals and the Ambiguous Welfare Effects of Forced Open Access: A Two-sided Model

Frank Mueller-Langer and Richard Watt

September 2013 


\section{Working Paper Series of the German Data Forum (RatSWD)}

The RatSWD Working Papers series was launched at the end of 2007. Since 2009, the series has been publishing exclusively conceptual and historical works dealing with the organization of the German statistical infrastructure and research infrastructure in the social, behavioral, and economic sciences. Papers that have appeared in the series deal primarily with the organization of Germany's official statistical system, government agency research, and academic research infrastructure, as well as directly with the work of the RatSWD. Papers addressing the aforementioned topics in other countries as well as supranational aspects are particularly welcome.

RatSWD Working Papers are non-exclusive, which means that there is nothing to prevent you from publishing your work in another venue as well: all papers can and should also appear in professionally, institutionally, and locally specialized journals. The RatSWD Working Papers are not available in bookstores but can be ordered online through the RatSWD.

In order to make the series more accessible to readers not fluent in German, the English section of the RatSWD Working Papers website presents only those papers published in English, while the the German section lists the complete contents of all issues in the series in chronological order.

Starting in 2009, some of the empirical research papers that originally appeared in the RatSWD Working Papers series will be published in the series RatSWD Research Notes.

The views expressed in the RatSWD Working Papers are exclusively the opinions of their authors and not those of the RatSWD.

The RatSWD Working Paper Series is edited by:

Chair of the RatSWD (2007/2008 Heike Solga; since 2009 Gert G. Wagner)

Managing Director of the RatSWD (Denis Huschka) 


\title{
Optimal Pricing and Quality of Academic Journals and the Ambiguous Welfare Effects of Forced Open Access: A Two-sided Model $^{1}$
}

\author{
Frank Mueller-Langer ${ }^{*}$ \\ Munich Center for Innovation and Entrepreneurship Research, Max Planck Institute for \\ Intellectual Property and Competition Law \\ Richard Watt ${ }^{*}$ \\ Department of Economics and Finance, University of Canterbury
}

${ }^{1}$ previously published as Munich Discussion Paper No. 2013-10 at http://epub.ub.uni-muenchen.de/16277/

\begin{abstract}
:
We analyse optimal pricing and quality of a monopolistic journal and the optimality of open access in a two-sided model. The predominant aspect of the model that determines the quality levels at which open access is optimal is the nature of the (non-linear) externalities between readers and authors in a journal. We show that there exist scenarios in which open access is a feature of highquality journals. Besides, we find that the removal of copyright (and thus forced open access) will likely increase both readership and authorship, will decrease journal profits, and may increase social welfare.
\end{abstract}

JEL Classification: L11, L82, O34

Keywords: Two-sided markets, academic journals, open access, removal of copyright, welfare effects

\footnotetext{
* This research benefitted from a Tilburg Law and Economics Center (TILEC) "Innovation, Intellectual Property and Competition Policy Grant". The authors are most grateful for this financial aid. We thank Ted Bergstrom, Mark McCabe, Ivan Png, Chris Snyder, Christian Zimmermann and participants of the NBER Workshop on Scholarly Communication, Open Science and Its Impact and the Tilburg Innovation, Intellectual Property and Competition Policy Conference for their valuable comments. We also thank participants of the Conference on Academia \& Publishing in Torino and the Third Workshop for Junior Researchers on the Law \& Economics of Intellectual Property and Competition Law as well as seminar participants in Barcelona, Christchurch and Madrid for helpful comments and suggestions.
} 


\section{Optimal Pricing and Quality of Academic Journals and the Ambiguous Welfare Effects of Forced Open Access: A Two-sided Model}

\section{Introduction}

Academic journals act as platforms upon which authors communicate their ideas to readers. Hence, journals need to attract both authors and readers in order to be able to provide their service. However, the interrelationship between authors and readers on the journal platform is more complex than a simple meeting place where ideas are exchanged. Readers attract authors to a journal, and authors attract readers to a journal, and both are attracted to higher-quality journals (Ellison, 2002; Heintzelman and Nocetti, 2009). Thus, the market for academic journals constitutes a twosided market (Filistrucchi, Geradin and van Damme, 2013; Parker and Van Alstyne, 2005; Rochet and Tirole, 2002 \& 2006; Rysman, 2009). Journal editors make decisions regarding readers and authors that are crucial to the final outcome of the quality that the journal achieves. Suppose that a journal acts to maximise profit. ${ }^{1}$ In such a scenario, the journal must decide on the subscription price for reader access, the author fee (submission and/or publication fees), and the overall quality of the journal, all with the objective of achieving maximal profit.

We study the following aspects of journal management. First, is it possible that one of the two prices (reader or author price) is optimally set to zero? Second, is a journal that does optimally set the reader price to zero (i.e. an "open-access" journal) more likely to be characterised by a lower quality level than a closed-access journal (i.e. one with a strictly positive subscription price for readers)? Finally, we analyse the effect of forced open access associated with the removal of copyright for academic works on author prices, journal quality and social welfare. ${ }^{2}$

These questions have important policy implications for the following reasons. The traditional subscription-fee-based publication model involves relatively low author prices but high reader prices. In contrast, the open-access model allows readers to access papers free of charge over the Internet and serves to increase the author price. However, the question whether open access can dominate the traditional publication model and be welfare-enhancing as well as sustainable in long-run equilibrium depends on the elasticities of demand on the author and reader side (McCabe and Snyder, $2005 \& 2007)$. In turn, the elasticity of author demand depends on the impact of open access on the quality of the journal, i.e. we take quality to be the expected number of citations per paper published (the expected impact factor). On the one hand, if open access leads to an increase in journal quality, author demand may be sufficiently inelastic to support high author prices that are "necessary for open access to be sustainable in long-run equilibrium" and high enough for the

\footnotetext{
${ }^{1}$ Other objectives may also be considered - the journal might act in order to maximise its impact factor, or it might act in order to maximise readership (diffusion of ideas published).

${ }^{2}$ We are interested in this aspect because of the recent, provocative paper by Stephen Shavell (2010) that advocates abolition of copyright in scientific publications. See also Towse, Handke and Stepan (2008) for a thorough overview of the economics literature on copyright law.
} 
open-access equilibrium to have positive welfare properties (McCabe and Snyder, 2013a, p. 3). On the other hand, if open access leads to a decrease in journal quality, the opposite result may hold true.

In the present paper, we model a journal as two-sided platform in order to account for some of the principal aspects of this complex market, i.e. optimal journal pricing, access to research outputs and journal quality (Bergstrom, 2001; Dewatripont et al., 2006; Gans, 2000; Jeon and Menicucci, 2006; McCabe, 2004). Thus, the paper adds to a relatively young body of literature that considers academic journals in two-sided markets (Jeon and Rochet, 2010; McCabe and Snyder, 2007; McCabe, Snyder and Fagin, 2013). It is related to the monopoly-platform model by Armstrong (2006). In contrast to Armstrong (2006), however, the platform's objective is to optimally choose its "quality" in addition to the optimal reader subscription price and author fee. Our paper is most closely related to McCabe and Snyder (2005), who also study the academic journal market by means of a two-sided market model. Similarly, we consider a profit-maximising, monopolistic journal $^{3}$ and investigate the question of how journal quality is related to open-access publishing. McCabe and Snyder (2005) find that the equilibrium reader price charged by a monopolistic journal is weakly increasing in journal quality, which implies that low-quality journals are more likely to adopt open access if an author's benefit of having her article read exceeds the reader's marginal effort and reading cost. However, they also argue that once this condition fails to hold the result will be reversed, i.e. higher-quality journals will favour open access. Our paper differs from McCabe and Snyder (2005) in two important aspects. First, it is the nature of the (non-linear) externalities between authors and readers of a journal rather than effort and distribution cost that determines whether open access is a feature of low or high-quality journals. Second, we treat journal quality differently. McCabe and Snyder (2005) assume that an increase in journal quality (editorial talent) has ceteris paribus a direct negative overall effect on authors as the acceptance rate decreases. Bad articles are identified as bad articles with a higher probability and thus are more likely to be rejected. In contrast, we assume that, on the quality range under study, authors' overall returns to choosing a journal increase in journal quality (Hamermesh, 1992; Heintzelman and Nocetti, 2009). Stated differently, the increase in academic prestige of a publication in a higher-quality journal more than outweighs the direct negative effects due to lower acceptance rates and longer response times (Oster, 1980). This assumption is further motivated by Ellison (2002), who suggests that the benefits from publishing in higher-quality journals, i.e. the rate at which papers published in the top-tier journals are cited relative to the rate at which papers published in the second-tier journals are cited, increased significantly from 1970 to 2000. It is in these two respects (non-linear externalities and positive overall returns of authors to choosing a higher-quality journal) that we believe our paper adds to McCabe and Snyder (2005) in order to address the before-mentioned countervailing effects that exist in the real world of academic publishing.

Our model can also be interpreted as a model of monopolistic competition if we consider ca-

\footnotetext{
${ }^{3}$ For instance, Bergstrom (2001, p. 190) suggests that "despite the possibility of new entrants into the industry and despite competition from nonprofit journals [...] the presence of potential competitors does not necessarily prevent monopoly pricing" due to a coordination failure within academia.
} 
pacity constraints on both sides of the market, i.e. that the number of readers and authors is limited. In this modified setup, we revisit the welfare effects of copyright protection and open access. The main structural assumptions in the model, which are linear demand and either linear or concave production functions, are relatively standard. We carry out an analysis based on numerical simulation.

We find several new results that add to the literature on two-sided journal markets and the economics of open access. ${ }^{4}$ First, if readers benefit less from the marginal author than authors do from the marginal reader, open access is rather a feature of high-quality journals. In contrast, if the diminishing returns lie on the other side of the market, open access is more likely to be a feature of low-quality journals. Second, we analyse the effects of a removal of copyright on journals, academics and social welfare. We find that removal of copyright (and thus forced open access) will likely increase both readership and authorship, will decrease journal profits, and may increase social welfare. It is in this respect that we believe our analysis is different from existing works such as Jeon and Rochet (2010) and McCabe and Snyder (2007).

In contrast to the existing literature, we have deliberately opted for a reduced-form model rather than a behavioural model based on individual utility maximisation. Thus, we eliminate the need for a host of simplifying assumptions at the individual level. Instead, any assumptions are made at the reduced-form level (i.e. assumptions on the actual demand and supply type relationships faced by journals, rather than assumptions on specific elements of individual behaviour). ${ }^{5}$ By using a reduced-form model, rather than sticking with the strongly behavioural models that currently populate the literature, we hope to provide a contrasting point of view, that is both interesting and valid, on the issue of optimal journal management.

\section{Model}

A "journal" is a set of papers. Papers are written by "authors" (and each author can submit at most one paper) and are consumed by "readers". Readers only have the option to purchase the entire set of papers in the journal, and cannot disaggregate the journal content for price reductions. Thus, the journal may be thought of as being a single volume (or several issues) of a particular title. The journal chooses quality, $q$, the price charged to readers, $p_{r}$, and the price charged to authors, $p_{a}$.

In this paper we treat "quality" as an exogenous choice variable of the journal. We neither specify or model how this choice is made, nor do we define exactly what the quality variable measures in terms of precise statistical information. In reality, quality is controlled by the journal via the referee process. Quality is taken to represent any given variable, under the control (either direct or indirect) of the journal, indicating to the academic community that the papers published have some guarantee of being novel, interesting and valuable. For example, we could take quality

\footnotetext{
${ }^{4}$ For a general discussion of open-access policy and the economics of open access, see Suber (2012).

${ }^{5}$ Note that assumptions at the individual behavioural level in the end all just condition the final reduced form of the model anyway. Thus they could always be restated as assumptions directly on the reduced form.
} 
to be the expected number of citations per paper published (the expected impact factor). Quality, then, is understood to be a measure of the perceived value of a given journal as an outlet for scientific information. The higher this quality, the more readers value reading the journal, and the more authors value publishing in it. ${ }^{6}$

Our treatment of quality reflects our reduced-form strategy - there is no need to specify how the journal chooses its quality. Once we take into account how this choice affects a journal's profits through the effects it has upon both readers and authors we only need to accept that each journal is able to choose its quality standard. All that is required is that quality is somehow chosen by the journal. Once that choice is made, it can be readily discerned by all market participants, and it is something that is desirable by both readers and authors. Considering the real world of academic publishing this seems reasonable as it is generally not difficult for market participants (readers and authors) to be able to rank journals according to their perceived quality, which may be thought of as a measure of the desirability of reading the journal, or of publishing in it. As long as a rank order of journals in terms of quality can be constructed, any measure that reflects that rank order works for our variable $q$. That is, for any $i$ and $j$, if journal $i$ is ordinally ranked ahead of journal $j$, then any two numbers $q_{i}>q_{j}$ can be used. Our assumption is simply that such a measure for this ranking exists, ${ }^{7}$ and is commonly observed by all participants.

The journal acts in order to maximise profits. Given the choice $\left(q, p_{r}, p_{a}\right)$, the number of readers that the journal attracts is endogenously given by $n_{r}\left(q, p_{r}, n_{a}\right)$, and the number of authors that are included in the subscription that is sold to readers is endogenously given by $n_{a}\left(q, p_{a}, n_{r}\right)$. Both the number of readers and the number of authors are determined in part by the quality chosen. The number of readers (authors) has a direct dependence on the price charged to readers (authors). The relationship between the number of readers and the price charged to authors, and between the number of authors and the price charged to readers, is indirect. The number of readers is (partially) determined by the number of authors, and vice versa. ${ }^{8}$ Since the number of authors published depends upon the choices made by the journal, this is a "long-run" analysis.

Here we can already see that the optimal pricing strategy of a monopoly journal is by no means transparent. It could happen that with low quality it is still optimal to charge a positive reader price but no author charge. In that way, for a given quality, the number of authors is large, which also gives value to readers, thereby compensating for the low quality variable. Likewise at high quality it may turn out to be optimal not to charge readers, and rather to charge authors a publishing fee

\footnotetext{
${ }^{6}$ For example, one might also want to interpret quality as being the monetary equivalent payoff to readers from reading, and also as the monetary equivalent payoff for authors in publishing. The greater $q$ is, the more valuable is the journal both to read and to publish in. Under such an interpretation, one would expect the number of readers to increase as the net monetary benefit from reading, $q-p_{r}$, increases (assuming it is positive), and the number of authors to increase as their net monetary benefit, $q-p_{a}$, increases (again assuming it is positive), both of which seem reasonable from a behavioural perspective, assuming of course that one does not run into a binding constraint on the actual supply of either readers or authors. We discuss the issue of binding "capacity" constraints below.

${ }^{7}$ Kóczy and Nichifor (2013) discuss a variety of popular ranking mechanisms, and suggest a particular ranking, along with the corresponding values of $q_{i}$, that satisfies a set of very reasonable axioms.

${ }^{8}$ The journal sells space to authors. Hence, the author price refers to the cost for an author to publish one paper. On the other hand, once the journal content is found, the subscription price to readers gives a reader access to all the papers in that issue of the journal.
} 
(this maximises readership, which in turn makes it more interesting for authors to publish). The optimal pricing strategy, and also the optimal choice of quality, will depend on the shapes of the two reduced-form equations $n_{r}$ and $n_{a}$, and the interrelationships between them.

Our assumptions reflect reality. Readers choose to read a journal depending on its content (which is given by the number of papers in it, $n_{a}$, and the quality of those papers, $q$ ), and the price charged to readers, $p_{r}$. Authors' willingness to publish in a journal depends on the quality of the journal, $q$, the audience reached, $n_{r}$, and the cost of publishing, $p_{a} \cdot{ }^{9}$ The fact that the two functions $n_{r}\left(q, p_{r}, n_{a}\right)$ and $n_{a}\left(q, p_{a}, n_{r}\right)$ are interdependent, i.e. the value of each depends (in part) upon the value of the other, captures the two-sided market feature of academic journals as platforms for readers and authors.

We can interpret the functions $n_{r}\left(q, p_{r}, n_{a}\right)$ and $n_{a}\left(q, p_{a}, n_{r}\right)$ in two different ways, both of which will be exploited in the paper. First, for given values of $q$ and $n_{a}$, say $\bar{q}$ and $\bar{n}_{a}$, we should understand $n_{r}\left(\bar{q}, p_{r}, \bar{n}_{a}\right)$ to be a demand function in the sense that it relates the price for reading to the number of readers who purchase. On the other hand, for given values of $q$ and $p_{r}$, say $\bar{q}$ and $\bar{p}_{r}$, we should understand $n_{r}\left(\bar{q}, \bar{p}_{r}, n_{a}\right)$ to be a production function, in the sense that papers (here, authors) are what attract readers to a journal. In the same way, $n_{a}\left(\bar{q}, p_{a}, \bar{n}_{r}\right)$ is again a demand function, and $n_{a}\left(\bar{q}, \bar{p}_{a}, n_{r}\right)$ is a production function (this time, reflecting the dependence of the number of authors that are attracted to a journal on the number of readers of that journal). For $i, j=r, a$ and $i \neq j$, we assume $\frac{\partial n_{i}}{\partial p_{i}}<0, \frac{\partial n_{i}}{\partial n_{j}}>0$ and $\frac{\partial^{2} n_{i}}{\partial n_{j}^{2}} \leq 0$. Thus, the demand functions are negatively sloped and the production functions are positively sloped and (weakly) concave.

We also assume $\frac{\partial n_{r}}{\partial q}>0$ and $\frac{\partial n_{a}}{\partial q}>0$. First, readers prefer higher-quality papers. Second, authors also prefer higher-quality journals. The latter assumption will only hold locally, on a range of quality levels. The higher the quality of a journal, the greater is the willingness of authors to supply papers to that journal (for CV effects and the fact that higher-quality journals are likely to reach a larger audience, and thus are more likely to be cited). But, the greater the quality hurdle, the lower is the acceptance rate. Thus, while a high-quality journal will have a larger set of papers to choose from, they are also more selective in their choosing. ${ }^{10}$ Almost certainly, the number of published papers is a non-monotone function of quality, since at some very high quality levels the quality filter will outweigh the effect of increased submissions. Indeed, we can even envisage that there could be a sufficiently high quality level such that no authors exist that can actually write a paper of such quality. ${ }^{11}$ However, throughout the paper, we assume that for the relevant range of quality levels there are more published authors as quality increases. This assumption is based upon observation of the real world of academic publishing. At least in the field of economics journals, those at the top of the quality ladder are typically able to publish many papers, while it is the

\footnotetext{
${ }^{9}$ For instance, $p_{a}$ is the sum of submission fee and publication fee.

${ }^{10}$ Note that we abstract from modeling the referee process, under which papers are screened for quality. Here, all that is important is to recognise that $n_{a}$ is the number of papers that end up being published, and that will be determined by the number of papers that are submitted (decreases with author price and increases with number of readers), and the quality of the journal. The assumption that the number of papers published increases with quality reflects the assumption that submissions of sufficient quality increase in quality.

${ }^{11}$ See, for example, the Journal of Universal Rejection (http://www.universalrejection.org/).
} 
journals of lower perceived quality that may struggle with finding papers to publish. Hence, it appears that the threshold quality level for authors to decline as quality increases has not been reached for any economics journal that we are aware of.

Our assumption that the number of published papers increases with quality is not innocuous to the results of our model. As we shall see, in the model we end up with profits being a strictly increasing function of quality, and thus each journal wants to increase quality as much as possible. However we should not interpret this result as implying that journals will set quality at an infinitely high level as we are only carrying out a local analysis in terms of quality. If we were to carry out a full consideration of the non-monotone functional relationship between the number of papers published and the journal's quality, there would exist a sufficiently high level of quality such that profits end up decreasing with quality as it becomes extremely difficult to find papers of sufficient quality to publish. In such a model there would be a finite optimal level of quality. ${ }^{12}$ We will re-address this assumption later on, once we have found the optimal quality-setting strategy for the journal.

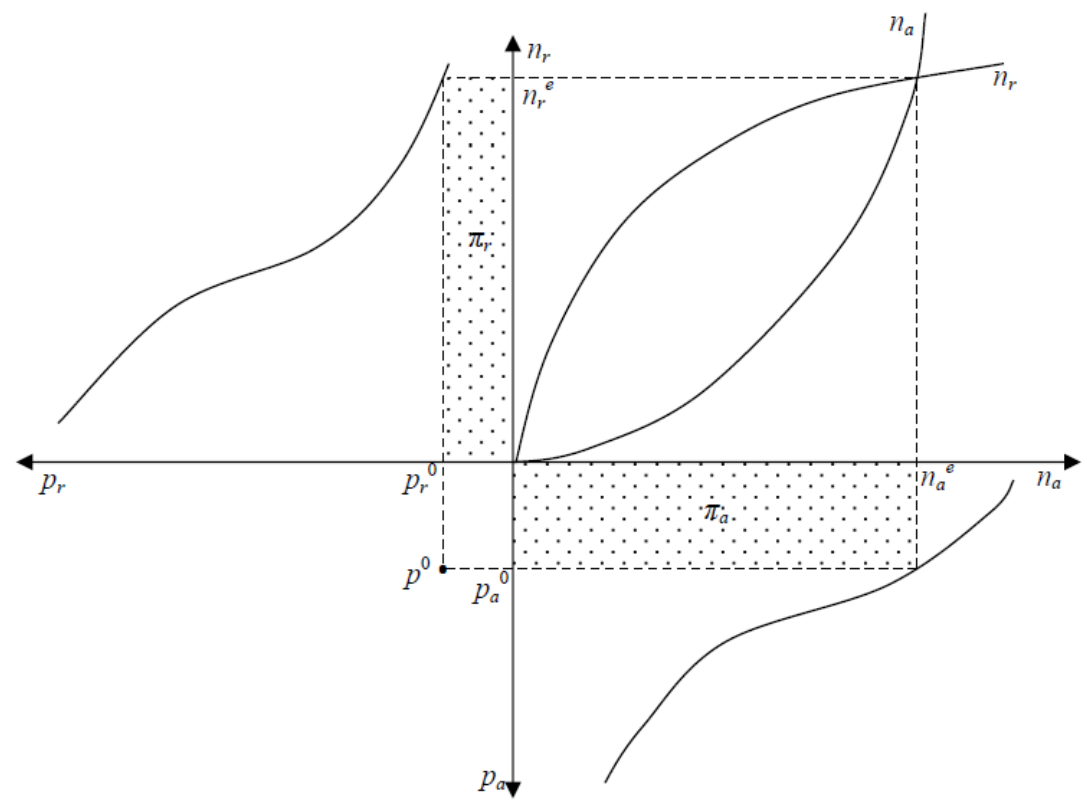

Figure 1: Two-sided market: demand and production functions and profit

Figure 1 illustrates both the demand curve aspect and the production function aspect of the journal platform, always taking quality to be fixed. The upper left-hand [lower right-hand] panel shows the demand curve aspect of $n_{r}\left(q, p_{r}, n_{a}\right)\left[n_{a}\left(q, p_{a}, n_{r}\right)\right]$, and the upper right-hand panel shows the production function aspects. Figure 1 highlights a very important aspect of the journals market. It is two-sided, and so the choice of reader price (a determinant of the number of readers) cannot be taken independently from the choice of author price (which is a determinant of the number of authors). There is only one consistent choice in this graph, which is labeled as point $p^{0}$ in the lower

\footnotetext{
${ }^{12}$ We are currently elaborating such a model.
} 
left-hand panel. Only with that choice of prices will the number of authors $\left(n_{a}^{e}\right)$ be consistent with the number of readers $\left(n_{r}^{e}\right)$, where the superscripts ${ }^{e}$ refer to endogenous equilibrium values.

Imagine that, from the situation drawn in Figure 1, the journal decided to increase the reader price while leaving the author price unchanged. Such an increase in the reader price will cause a shift along the demand curve for the number of readers, thus reducing $n_{r}$. The production function for readers will itself shift, since it is parameterised by the reader price. Since we assume that the number of readers is a decreasing function of the price for reading, the production function will shift downwards. There is a resulting shift along the production function for the number of authors. Next, the demand function for the number of authors is parameterised by the number of readers. The number of readers has decreased, which will shift the demand function for the number of authors inwards. Finally, the number of authors has also been decreased, which will shift the demand function for the number of readers inwards. These shifts will continue until a new equilibrium point is attained. We assume throughout that the equilibrium process just described is stable, in the sense that for any $\left(q, p_{r}, p_{a}\right)$, the curves adjust such that there is a mutually compatible pair $\left(n_{r}, n_{a}\right)$.

\subsection{Profit-maximising decisions}

Following Jeon and Rochet (2010), we study the case of an online journal, rather than a journal that publishes in hard-print format. This simplifies the analysis as it allows us to realistically assume that the marginal cost of supplying readers is zero. ${ }^{13}$ This assumption is a first approximation for the changing conditions under which an online-only journal supplies its content in the digital era for the following reasons. First, as McCabe and Snyder (2013a, p. 1) put it, "the advent of the Internet [...] effectively reduces the cost of distributing the journal to readers close to zero". Second, authors typically produce and format the electronic files and data themselves (Hilty, 2005). At least in economics, authors usually follow precise author guidelines and use the style files and templates provided by the journal, which thereby significantly reduces the cost of processing of accepted papers, i.e. copyediting and typesetting. Third, for submission and peer-review, commercial publishers frequently use cost-effective web-based editorial end-to-end systems that significantly reduce the journal's cost of handling a submitted article and processing an author's account. Fourth, the referee process is typically free of charge for the publishers (Hilty, 2005). The profits earned by the journal are $\pi\left(q, p_{r}, p_{a}\right)=p_{r} \cdot n_{r}+p_{a} \cdot n_{a}=\pi_{r}+\pi_{a}$.

Figure 1 illustrates the profits derived from the reader side of the market $\left(\pi_{r}\right)$ and the author side of the market $\left(\pi_{a}\right)$. The sum of these two rectangular areas is the total profit. Note that a unilateral increase of the reader price decreases the profit in the author market since the author price stays constant and the number of authors decreases. In addition, it may either increase or decrease the profit in the reader market (it goes from a tall thin rectangle to a shorter but wider one).

\footnotetext{
${ }^{13}$ For simplicity, we also assume the fixed costs of the journal to be zero. Fixed costs can easily be incorporated. However, they will have no effect on the model, except for giving a shut-down condition.
} 
The journal chooses $\left(q, p_{r}, p_{a}\right)$ in order to maximise its profits. We model this recursively. First, hold quality fixed at some level, $q$. Given that quality, we analyse the optimal pricing policy of the journal, $p^{*}(q)=\left(p_{r}^{*}(q), p_{a}^{*}(q)\right)$. Considering these optimal prices for each quality level, we derive the optimal quality that the journal should choose.

Firstly, for any given $\left(q, p_{r}, p_{a}\right)$ it is necessary to simultaneously solve the two equations $n_{r}\left(q, p_{r}, n_{a}\right)$ and $n_{a}\left(q, p_{a}, n_{r}\right)$ for the two equilibrium levels of readers and authors, $n_{r}^{e}\left(q, p_{r}, p_{a}\right)$ and $n_{a}^{e}\left(q, p_{r}, p_{a}\right)$. The profit of the journal is $\pi\left(q, p_{r}, p_{a}\right)=p_{r} \times n_{r}^{e}\left(q, p_{r}, p_{a}\right)+p_{a} \times n_{a}^{e}\left(q, p_{a}, p_{r}\right)$. The derivatives with respect to the two prices are given by $\frac{\partial \pi}{\partial p_{i}}=n_{i}^{e}+p_{i} \frac{\partial n_{i}^{e}}{\partial p_{i}}+p_{j} \frac{\partial n_{j}^{e}}{\partial p_{i}}$, where $i, j=r, a$ and $i \neq j$. Carrying out the implied second derivatives, it turns out that a sufficient condition for profits to be concave in the price $p_{i}$ is $\frac{\partial^{2} n_{i}}{\partial p_{i}^{2}} \leq 0$ and $\frac{\partial^{2} n_{i}}{\partial n_{j} \partial p_{i}} \geq 0$. Assuming concavity, the two first-order conditions for optimal choices of the two prices are $\frac{\partial \pi}{\partial p_{r}}=0$ and $\frac{\partial \pi}{\partial p_{a}}=0$. The simultaneous solution gives us the two optimal prices as functions of the quality, $p_{r}^{*}(q)$ and $p_{a}^{*}(q)$. The indirect profit function is then given by

$$
\pi(q)=p_{r}^{*}(q) \times n_{r}^{e}\left(q, p_{r}^{*}(q), p_{a}^{*}(q)\right)+p_{a}^{*}(q) \times n_{a}^{e}\left(q, p_{r}^{*}(q), p_{a}^{*}(q)\right) .
$$

This function is maximised with respect to $q$.

\section{$2.2 \quad$ A simplified model}

In order to see how the model works, we assume three different scenarios, each of which is characterised by linear demand functions for both readers and authors. They differ with respect to the degree of concavity of the two production functions. Specifically, in scenario 1 we assume that both production functions are affected by diminishing returns (i.e. they are both concave). In scenario 2 the production of readers, taking authors as an input, has diminishing returns (i.e. is concave) while the production of authors, taking readers as the input, is linear. In scenario 3 the reader production function is linear and the author production function is concave.

In each of the three scenarios, demand is given by a linear form, with vertical intercept (i.e. maximum feasible price) equal to $\alpha q \cdot{ }^{14}$ Thus, greater levels of quality correspond to parallel shifts of the two demand curves. We have no reason to assume that the effect of a marginal change in quality upon the demand curve of readers is any different than the same effect for authors. So in the interest of keeping our model as uncluttered as possible, we assume that this effect is equal for both sides of the market $(\alpha) .^{15}$

\footnotetext{
${ }^{14}$ Our linear reader (author) demand equation is a function of quality, but not a function of the number of authors (readers), for the following reason. In the real world of academic publishing journals do not commit to a given number of authors (readers), but there is a commitment to quality. The reader (author) price is charged based on quality, not the thickness (readership) of the journal.

${ }^{15}$ The assumption of linear demand is, of course, only intended as a first approximation to any real-life scenario. Non-linear forms increase the complexity of the model enormously, with no qualitative change in the results that are obtained. Basically the linear form is the least complex way in which we can assure that when there are no readers, $n_{r}=0$, then no authors are attracted to the journal, so that $n_{a}=0$. Likewise, no authors implies no readers. This feature can also be incorporated into non-linear demand forms, but as stated above, this leads to significant analytical complexity with no real gain in the model's output.
} 


\subsubsection{Scenario 1: Diminishing returns on both sides}

Here, we assume $n_{r}=\sqrt{n_{a}}\left(\alpha q-p_{r}\right)$ and $n_{a}=\sqrt{n_{r}}\left(\alpha q-p_{a}\right)$, which we rewrite as

$$
\begin{aligned}
& n_{r}=\sqrt{n_{a}} \beta_{r}, \\
& n_{a}=\sqrt{n_{r}} \beta_{a},
\end{aligned}
$$

where $\beta_{i} \equiv \alpha q-p_{i}$ for $i=r, a$. Recall that both of $n_{r}$ and $n_{a}$ are constrained to be positive, so we are restricted to parameter values such that $\beta_{i}>0$ for $i=r, a$. That is, we can only consider prices that satisfy $p_{i}<\alpha q$ for $i=r, a$.

It is easy to show that the solution to the two equations (1) and (2), outside of the trivial solution at $(0,0)$, lies at $n_{r}=\left(\beta_{r}^{4} \beta_{a}^{2}\right)^{\frac{1}{3}}$ and $n_{a}=\left(\beta_{r}^{2} \beta_{a}^{4}\right)^{\frac{1}{3}}$. The profits of the journal are:

$$
\pi=p_{r} n_{r}+p_{a} n_{a}=p_{r}\left(\beta_{r}^{4} \beta_{a}^{2}\right)^{\frac{1}{3}}+p_{a}\left(\beta_{r}^{2} \beta_{a}^{4}\right)^{\frac{1}{3}}
$$

The profit function is perfectly symmetric in the two prices. That is, the function is of the form $\pi=f\left(p_{r}, p_{a}\right)+f\left(p_{a}, p_{r}\right)$, where $f(x, y) \equiv x\left((\alpha q-x)^{4}(\alpha q-y)^{2}\right)^{\frac{1}{3}}$. Thus, it makes no difference to the problem how we label our price variables. In the optimal solution it must be true that $p_{r}^{*}=p_{a}^{*}$. We can use this insight to help us solve the maximisation problem. We add the restriction $p=p_{r}=p_{a}$ to the existing restrictions $p_{i}<\alpha q$ for $i=r, a$. Substituting this first restriction into the objective function gives $\pi=2 p\left(\beta^{4} \beta^{2}\right)^{\frac{1}{3}}=2 p\left(\beta^{6}\right)^{\frac{1}{3}}=2 p \beta^{2}$. Here, $\beta=\alpha q-p$, so that $\frac{\partial \beta}{\partial p}=-1$. The first-order condition for an optimal solution is $\frac{\partial \pi}{\partial p}=0 \Rightarrow 2 \beta^{* 2}-4 p^{*} \beta^{*}=0$, where $\beta^{*}=\left(\alpha q-p^{*}\right)>0$. This equation can be expressed as $p^{*}=\frac{\alpha q}{3}$. The second-order condition on this maximisation problem is $-8 \beta+4 p<0$, which is $-8 \alpha q+12 p<0$. At the stationary point (which is unique on the range $p<\alpha q$ ), we have $-8 \alpha q+12 p^{*}=-8 \alpha q+4 \alpha q=-4 \alpha q<0$. Thus, the second-order condition is satisfied at the optimal solution. In short, the two optimal prices for scenario 1 are identical linear functions of quality:

$$
p_{r}^{*}=p_{a}^{*}=\frac{\alpha q}{3} .
$$

Intuitively, the fact that both sides of the journal market are symmetric drives this result. Authors benefit from the marginal reader in the same way that readers benefit from the marginal author. In this case, it is optimal for the journal to increase the reader price as well as the author price if quality increases.

\subsubsection{Scenario 2: Diminishing returns to authors}

We now assume (i) $n_{r}=\sqrt{n_{a}}\left(\alpha q-p_{r}\right)$ and (ii) $n_{a}=n_{r}\left(\alpha q-p_{a}\right)$. (i) An increase in the total number of published articles ceteris paribus induces an increase in the number of readers in a decreasing way. (ii) Intuitively, authors' benefit per article is linear in the number of readers as brought forward by McCabe and Snyder (2005). In this case, readers benefit less from the marginal 
author than authors do from the marginal reader. In Appendix A, we show that the optimal prices in this scenario are:

$$
\begin{aligned}
& p_{a}^{*}=\frac{5 \alpha q-\sqrt{4 \alpha^{2} q^{2}+7 \alpha q}}{7}, \\
& p_{r}^{*}=\frac{21 \alpha q-4 \alpha^{2} q^{2}-2 \alpha q \sqrt{4 \alpha^{2} q^{2}+7 \alpha q}}{49} .
\end{aligned}
$$

The two optimal prices are graphed in Figure $2 .{ }^{16}$ For relatively high quality levels, the optimal reader price is decreasing in quality. This result is consistent with the result brought forward by McCabe and Snyder (2005) in their scenario where high-quality journals are more likely to adopt open access. In contrast, however, if quality is relatively low, we find that the optimal reader price is increasing in quality. Note that it is not possible for the journal to pay readers, that is, the reader price cannot be negative. In reality, the optimal reader price equation (4) dictates negative reader prices for all quality levels exceeding $q_{0}$, the strictly positive solution to $p_{r}^{*}\left(q_{0}\right)=0$. Figure 2 depicts these negative prices as a dashed curve. Since it is not realistically feasible to pay readers, ${ }^{17}$ on that range of quality levels the journal would be restricted to the corner solution with $p_{r}^{*}=0$, indicated by the continuation of the solid curve along the axis. Thus, the optimal reader price is a piecewise function.

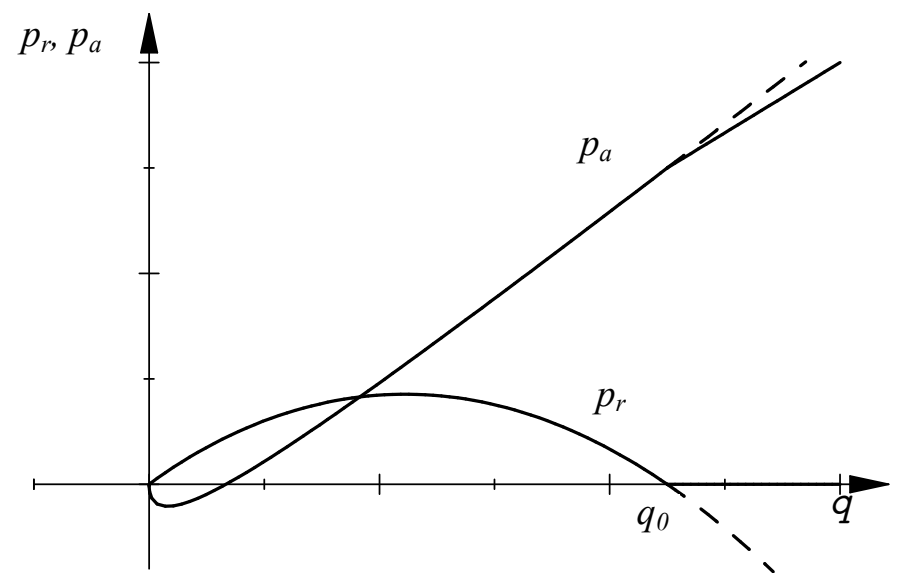

Figure 2: Optimal prices under scenario 2

This also affects the optimal author price. When the reader price is restricted to 0 , the optimal author price is no longer given by equation (3). Instead, above $q_{0}$, the optimal author price is linear and equal to $\frac{\alpha q}{3} .{ }^{18}$ Thus, the author price graph in Figure 2 is also piecewise, as can be seen by the

\footnotetext{
${ }^{16}$ In our simulations, we used $\alpha=1$, although it is relatively simple to see that taking any other (positive) value would not alter the shapes of the graphs obtained, but only their values.

${ }^{17}$ Most notably, however, Jeon and Rochet (2010) find that a social planner would subsidise the marginal reader and choose a strictly negative reader price under the first-best outcome.

${ }^{18}$ See Appendix B.
} 
kink in the optimal author price graph at $q_{0}$. The dashed curve is the continuation of the optimal author price, which would assume that negative reader prices are feasible.

The intuition behind our results is the following. Since authors benefit more from the marginal reader, i.e. for high quality levels and increasing quality, it is optimal for the journal to decrease the reader price to maintain or even increase readership. This in turn increases profits indirectly due to its impact on the more valuable author population.

\subsubsection{Scenario 3: Diminishing returns to readers}

Our third scenario is the opposite of scenario 2. Specifically, in scenario 3 we assume (i) $n_{r}=$ $n_{a}\left(\alpha q-p_{r}\right)$ and (ii) $n_{a}=\sqrt{n_{r}}\left(\alpha q-p_{a}\right)$. (i) Intuitively, readers' benefit per article is linear in the number of articles (authors). (ii) An increase in the total number of readers ceteris paribus induces an increase in the number of published articles in a decreasing way. In this case, authors benefit less from the marginal reader than readers do from the marginal author. Given the symmetry between scenarios 2 and 3, it is straight-forward to see that the solution will be exactly opposite to that in scenario 2 , i.e.

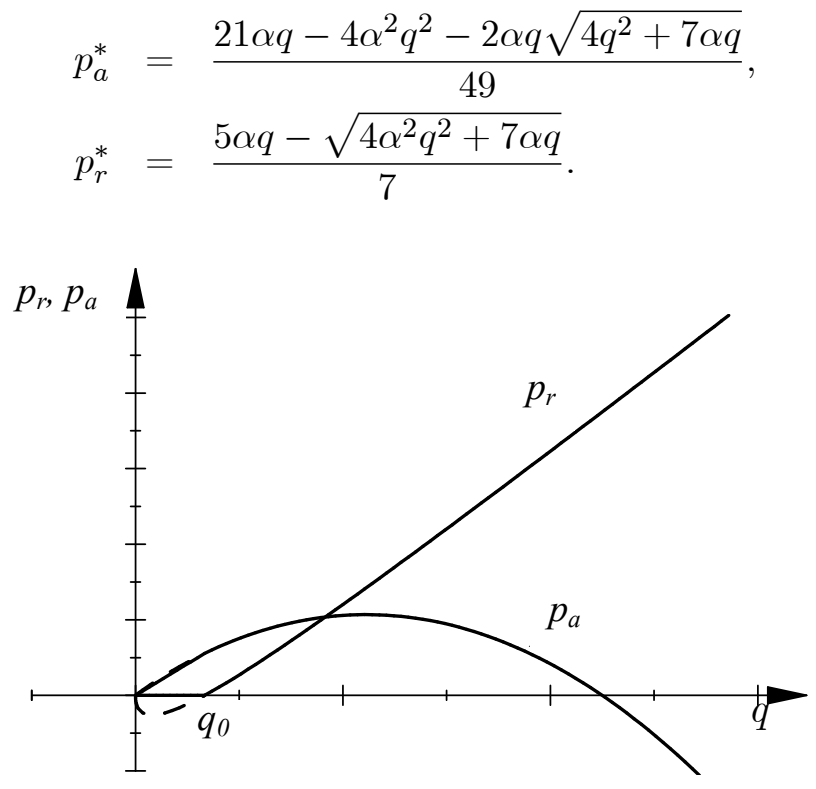

Figure 3: Optimal prices under scenario 3

The same comments as for Figure 2 apply, but now the zone of qualities for which the reader price is set to 0 as a corner solution is $q<q_{0}$, where $q_{0}$ is the positive solution to $p_{r}^{*}(q)=0$. On this zone, again $p_{a}^{*}(q)=\frac{\alpha q}{3} \cdot{ }^{19}$

Intuitively, since readers benefit more from the marginal author, i.e. for high quality levels and increasing quality, it is optimal for the journal to decrease the author price to maintain or even

\footnotetext{
${ }^{19}$ See Appendix B.
} 
increase the population of authors. This in turn increases profits indirectly due to its impact on the more valuable reader population.

\subsection{Discussion}

Our simulations serve to show a couple of important points as regards pricing. First, the question which of the production technologies has the decreasing returns seems to be crucial. The diminishing returns to authors (readers) drive the non-monotonicity of the relationship between the reader (author) price and quality in scenario 2 (3). In contrast, when both the production of readers using authors as an input and the production of authors using readers as an input are concave production processes (scenario 1), the resulting optimal author and reader pricing functions turn out to be linear functions of quality. Hence, our simulation points to there being no quality levels for which either price goes to zero. Thus, in that scenario, there is no scope for open access as an optimal pricing strategy. On the other hand, when the production of readers has decreasing returns to the addition of authors, but the production of authors is linear in readers (scenario 2), then our simulation reveals that open access is the optimal choice for the journal (i.e. to charge readers a price of zero) if the quality of the journal is relatively high (Figure 2). Thus, in this scenario, open access is a feature of high-quality, rather than low-quality, journals. Third, when it is the author production process that has decreasing returns to the addition of readers, and the reader production function is linear, we get the opposite result; open access is a feature of optimal journal pricing only for very low-quality journals. We can think of logical reasons to support either scenario. Intuitively, even though additional authors are non-perfect substitutes for producing readers, each reader has a strict time budget constraint for reading papers. Thus, adding papers is the addition of a variable (albeit non-perfectly substitutable) input to a fixed time input. This would imply diminishing returns and make scenario 2 realistic. On the other hand, one may realistically assume that authors have a fixed time budget for writing papers. For instance, teaching as well as grant

writing and other administrative tasks may set an upper bound for the fixed time budget for writing papers. In this case, the addition of readers into the author production function is the addition of new units of perfectly substitutable inputs to a fixed time input, which traditionally would be thought to involve diminishing returns. For this reason, scenario 3 may be realistic. Besides, our simulations reveal that there is scope for negative author prices in two of our scenarios, something that is rather rare in the real world of journal management. In scenario 2, we get very low-quality journals having to pay authors in order to attract them to publish in the journal. In scenario 3 , very high-quality journals pay their authors.

\subsection{Comparative analysis}

We now compare the three above scenarios graphically. Specifically, we look at the level of profit obtained, the level of social welfare, and the share of total social welfare that is retained by academics (readers and authors), all as functions of $q$. 
Above, we have already determined the optimal prices in each of the three scenarios. The remaining graphs are all derived from those optimal prices. The easiest way to understand the actual equations involved is to recall that the equilibrium numbers of both authors and readers, $n_{r}^{*}$ and $n_{a}^{*}$, are both functions of the two optimal prices. Since the two optimal prices are both functions of quality $q, n_{r}^{*}$ and $n_{a}^{*}$ are also functions of quality. Hence, for either scenario, the equilibrium level of profits is given by $\pi(q)=p_{r}^{*}(q) n_{r}^{*}(q)+p_{a}^{*}(q) n_{a}^{*}(q)$. To calculate welfare, we look at the surplus retained by academics (readers and authors) plus journal profits. To consider the welfare of academics, we use the well-known concept of consumer surplus. Our demand curves for the journal's services by both readers and authors are linear, thus "consumer surplus" on each side of the market is a triangle. Since our demand curves are $n_{i}=g\left(n_{j}\right)\left(\alpha q-p_{i}\right)$, for $i, j=1,2$ and $i \neq j$, where $g(n)$ is either $\sqrt{n}$ or $n$, depending on the scenario, the vertical intercept (i.e. the price at which quantity goes to 0$)$ is $\alpha q$. The area of the triangle on side $i$ of the market is $C S_{i}(q)=\frac{1}{2} n_{i}^{*}(q)\left(\alpha q-p_{i}^{*}(q)\right)$ with $i=r, a$. Given this, total welfare is given by $W(q)=C S_{r}(q)+C S_{a}(q)+\pi(q)$ and the share of welfare that is retained by academics is given by $S(q)=\frac{C S_{r}(q)+C S_{a}(q)}{W(q)}$.

Table 1 depicts the graphs of the principal variables of the three scenarios. ${ }^{20}$ Recall that in scenarios 2 and 3, an unrestricted analysis would set negative reader prices for some ranges of quality. This is not realistically feasible. Thus, in reality, the reader price would be set to 0 . This has been taken into account in all of the graphs that appear in Table 1. For all of the following simulations, we have used $\alpha=1$.

For all scenarios, the level of profit that the journal earns is strictly increasing in quality. Thus journal managers will always strive to increase the perceived quality of their publication. This result is critically dependent upon two assumptions in our model - the assumption that the number of authors will increase with quality, and our implicit assumption that there is a neverending supply of both authors and readers. Recall that casual observation of the real world of journal publishing suggests that for all journals currently being produced, both assumptions are indeed valid. However, it is worthwhile to think about what would happen if one or the other assumption were to be violated. First, as already mentioned above, if the quality hurdle to publish increases sufficiently, then at some point the number of authors will begin to decrease as quality continues to increase. This implies that there will exist an optimal level of quality beyond which the journal will no longer wish to increase quality. Second, it may be more realistic to consider the possibility that as quality increases, and more and more readers and authors are added to the journal, the available population of one or the other is exhausted at some point. In essence, journals will, at some point, run into a capacity constraint on either authors or readers, that then will determine the exact level of quality that their journal attains. In that way, our model can also be interpreted as one of monopolistic competition, where the entire population of, say, authors is divided into mutually exclusive subsets, one for each journal. The quality of the journal is then determined by when their allocated number of authors is reached. To model the details of this

\footnotetext{
${ }^{20}$ All of the graphs have been generated using the MuPAD 3 package in Scientific Workplace, and they have also all been independently checked using Mathematica. All of the working behind the actual graphs was also done by hand. Details are available from the authors upon request.
} 
process is left for future research. We will revisit the issue of capacity constraints again below in our analysis of the effects of removal of copyright.

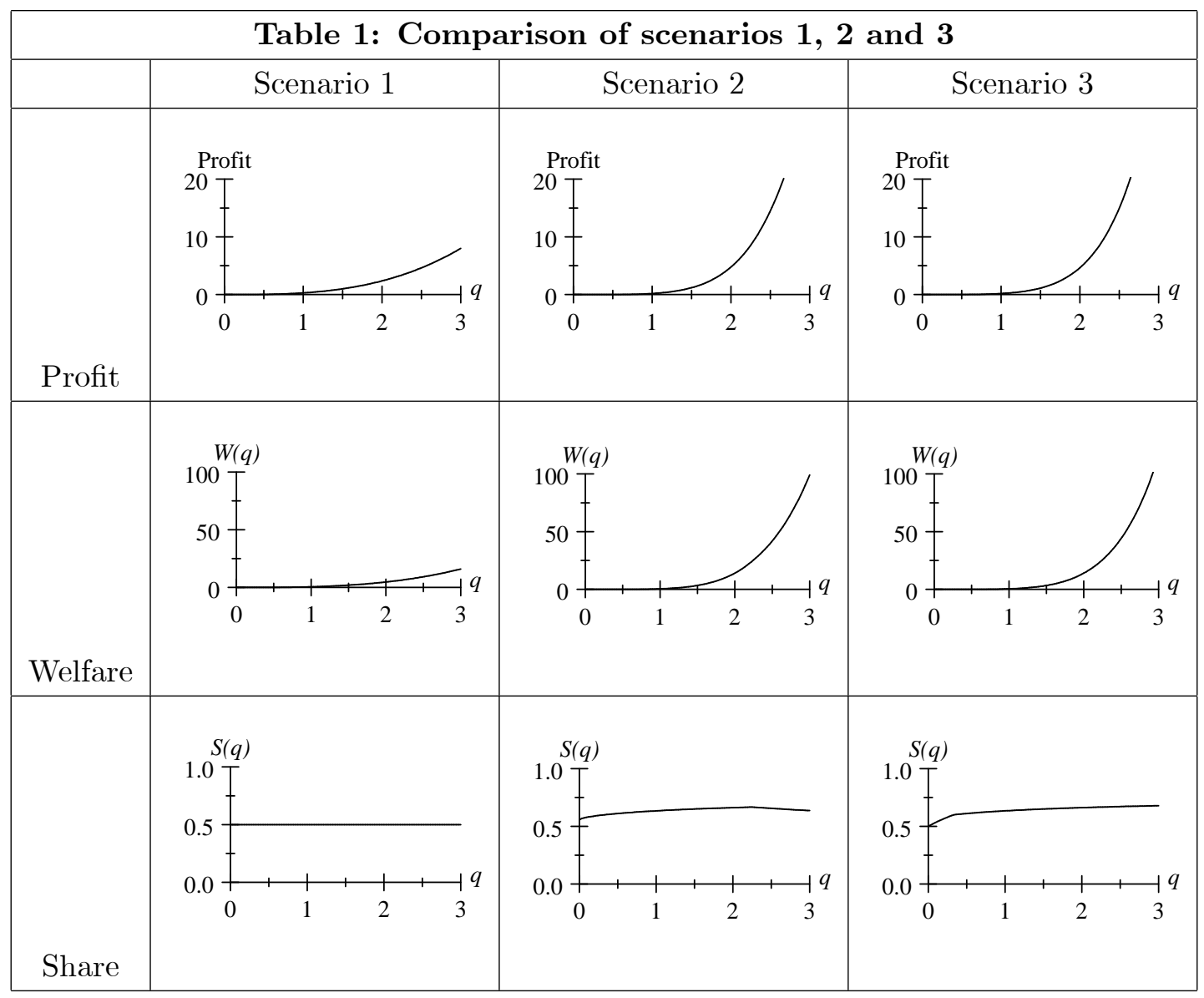

Social welfare, defined as the total sum of consumer surplus on both sides of the market plus journal profits, is strictly increasing in journal quality. Thus, the greater the level of quality that a journal can attain, the higher is the level of social welfare. However, the way that welfare is shared among the market participants is critically dependent upon the modelling assumptions. In our scenario 1 (diminishing returns on both production functions), the academics and the journal share welfare equally regardless of the quality of the journal. In the other two scenarios, the share of total surplus that is retained by readers and authors will lie within lower and upper limits. As quality increases, the share of welfare retained by individuals increases, but is never greater than 0.67 in scenario 2 and 0.75 in scenario 3. On the other hand, it never falls below 0.5 in both scenario 2 and 3. That is, in those two scenarios the readers and authors in aggregate always retain a strictly larger share of total surplus than the journal (as long as quality is strictly positive).

Consider Table 1. The piecewise element has the greatest effect on the share of academics' welfare in total welfare. In Figures 4 (scenario 2) and 5 (scenario 3) we show larger versions of these two graphs. Notice that, in scenario 2, the share of academic welfare in total welfare is 
increasing up to the point at which the reader price goes to zero, and is decreasing after that. The dashed line indicates where this share would go if it were feasible to pay readers. In Figure 5 we can see the detailed graph of academic welfare as a fraction of total welfare in scenario 3 . In scenario 3 , the share of academic welfare in total welfare is always increasing, but it is lower than it would be if readers could be paid on the section of the graph for which the journal is open access.

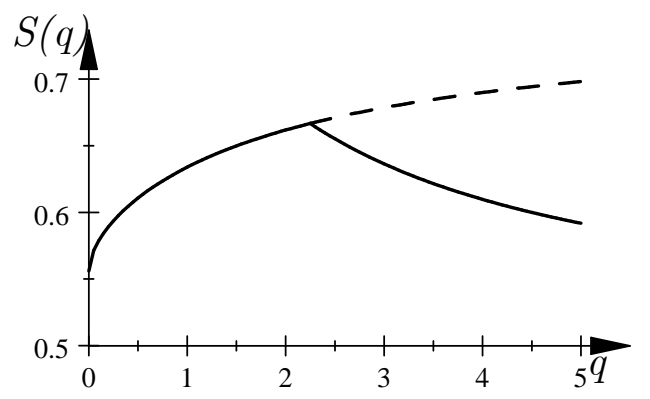

Figure 4: Share of academic in total welfare, scenario 2

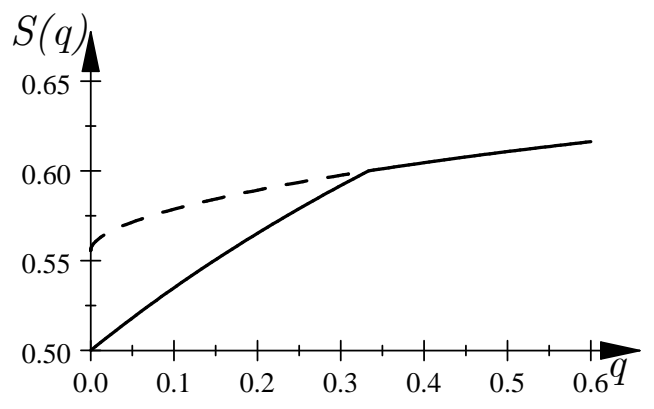

Figure 5: Share of academic in total welfare, scenario 3

\section{The effects of removal of copyright}

We can analyse the issue of copyright by simply noting that when there is copyright protection in place, the journal can act in the market for readers as a monopolist, while if there is no copyright, then the journal is far more open to competition from other publishers (including authors' own websites etc.). Thus, assume that the scenarios analysed above are those corresponding to the existence of copyright protection, and that when copyright protection is lifted (Shavell, 2010), the journal can no longer choose the reader price, which is then fixed at 0 . This simplifies the model significantly.

Now, the profit that the journal earns is equal only to what it can earn from authors. In Appendix B, we show that the optimal author prices when copyright is removed are $p_{a}^{*}=\frac{3 \alpha q}{7}$ under scenario $1, p_{a}^{*}=\frac{\alpha q}{3}$ under scenario 2 and $p_{a}^{*}=\frac{\alpha q}{3}$ under scenario 3 , respectively. By comparing these prices with the optimal author prices under copyright we can see that the removal of copyright serves to increase the optimal author price in all three scenarios. Most notably, high author fees are necessary for open access to be a sustainable long-run equilibrium and socially beneficial (McCabe and Snyder, 2013b). ${ }^{21}$ In contrast to the case of copyright protection, now the optimal author prices in scenarios 2 and 3 are strictly positive, and linear, for all levels of quality.

We now compare the three scenarios both with and without copyright. We evaluate our results in absolute and relative terms. ${ }^{22}$ First, we look at the relative comparisons, i.e. we are interested

\footnotetext{
${ }^{21}$ Note, however, that McCabe and Snyder (2013b) find no empirical support for a significant, positive effect of online access on citations in the economics and business literature.

${ }^{22}$ The absolute values of our variables would be altered by simply changing, for example, our assumption on the value of $\alpha$. However, as we shall see, the absolute value comparison, given $\alpha$, is still interesting.
} 
in the variable $z(q)$, where $z$ can represent the optimal author price, profits, welfare, or share of welfare. Let $z(q)_{c}$ be the value of $z$ under a regime of copyright protection, and let $z(q)_{n c}$ be its value when copyright is removed. Then we are interested in the relative change in $z$ from the removal of copyright, $\frac{z(q)_{n c}-z(q)_{c}}{z(q)_{c}}$. It turns out that in scenario 1, all of the relative changes are independent of the level of quality, and thus can be given as a specific percentage change. In the other scenarios, the relative effect from removal of copyright differs as quality changes. In Table 2 , all of the graphs shown are piecewise, since even under copyright, the inability to pay readers implies that for the ranges of quality when it would be optimal to pay readers, the reader price must be set at 0 . Thus, the removal of copyright has no effects at all on those zones of quality. We can now see that there are some significant differences between scenarios 2 and 3 . While in all scenarios, the journal loses profit when copyright is removed (on the zone for which they would like to charge a positive reader price), the percentage loss in profit is decreasing in scenario 2 and increasing in scenario 3. In scenario 2, the higher the level of quality of the journal, the smaller is the percentage loss in profits when copyright is removed. The opposite holds true for scenario 3.

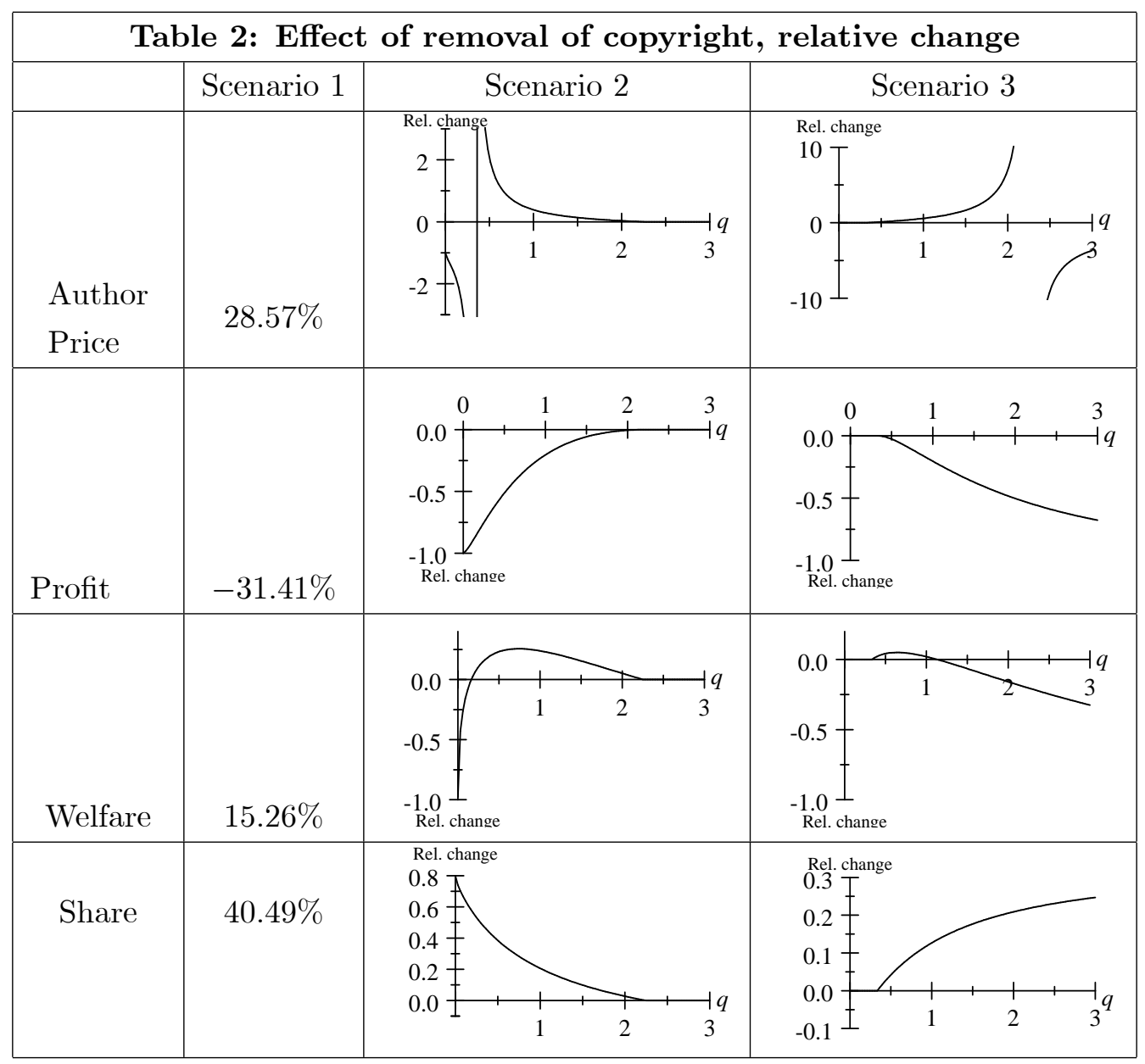

Now we analyse the absolute loss in profits in scenarios 2 and 3. In Figures 6 and 7 we show the absolute change in profits for these two scenarios. The important thing to notice about Figures 
6 and 7 is the huge difference in the scale of the vertical axis. While the levels of profit attained are the same under copyright in both scenarios (see Table 1, row 1), the removal of copyright in scenario 2 results in a relatively small absolute loss in profits at all quality levels (outside of those for which the reader price under copyright would be set at 0 ). In contrast, in scenario 3 it results in a similarly small loss for small levels of quality (below about $q=1.2$ ), but very large absolute loss in profits for high-quality journals. While the relative effect upon journal profit in scenario 2 is seemingly large for lower levels of quality, these losses are for very low levels of profit anyway. Removal of copyright in scenario 2 hardly affects the profits of journals at any quality levels. However, removal of copyright leads to large profit losses when profits are large in scenario 3 , a much more devastating result. If, for example, journals did have some fixed costs of operation (as is likely in the real world), then removal of copyright would only lead to the closure of some very low-quality journals in scenario 2 , but it can lead to the closure of high-quality journals in scenario 3. Hence, the removal of copyright as suggested by Shavell (2010) may be a rather dangerous strategy in a scenario 3 world.

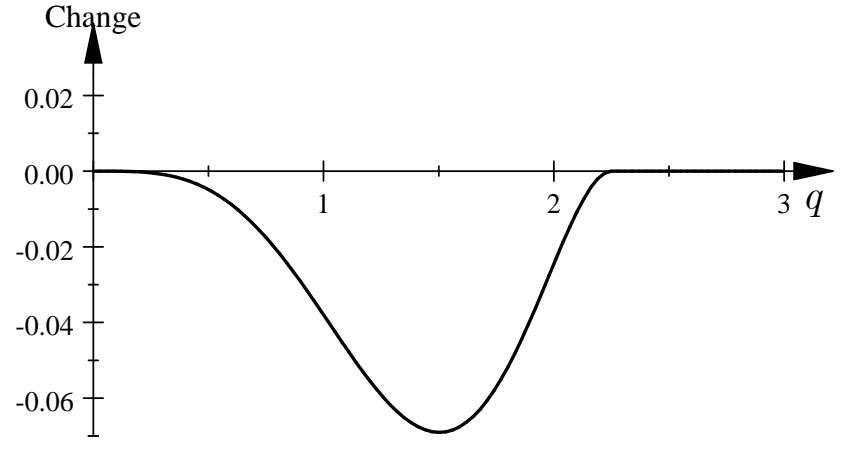

Figure 6: Absolute change in profit from removal of copyright; scenario 2

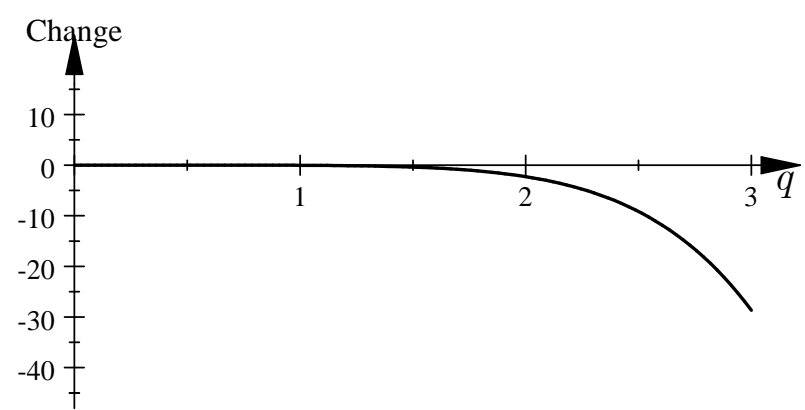

Figure 7: Absolute change in profit from removal of copyright; scenario 3

Regarding welfare, in scenario 2 there is a rather large zone of positive welfare gains in percentage terms, whereas in scenario 3 the zone of welfare gains is much smaller, and the relative gains are also smaller. Thus, assuming that social welfare is the policy objective, it would appear that removal of copyright might be a reasonable policy in scenario 2 , but not in scenario 3 . This intuition can again be confirmed by looking at the absolute changes in welfare from removal of copyright in Figures 8 and 9. Again we need to look at the scale of the vertical axis. In scenario 2 (Figure 8), while there is a very small negative part of the graph ${ }^{23}$ at levels of quality below 0.2 , the scale of these losses is totally insignificant compared to the gains at higher quality levels. In short, in scenario 2 removal of copyright leads to hardly any danger of welfare loss, and relatively interesting (upwards of about $20 \%$ ) welfare gains for almost all levels of quality. On the other hand, consider the absolute welfare

\footnotetext{
${ }^{23}$ Indeed, the negative section of the graph cannot even be discerned unless the vertical scale is changed by a factor of about $\frac{1}{100}$.
} 
change in scenario 3 (Figure 9). In this graph there is a positive section between levels of quality of $\frac{1}{3}$ and about 1.1. ${ }^{24}$ The rest of the graph lies below the horizontal axis, and at relatively large numbers, which implies that the removal of copyright leads to large welfare losses for those levels of quality. Thus, in scenario 3 the removal of copyright can improve welfare for low levels of quality but the improvement is miniscule, whereas for higher levels of quality, the change in social welfare is negative and significant.

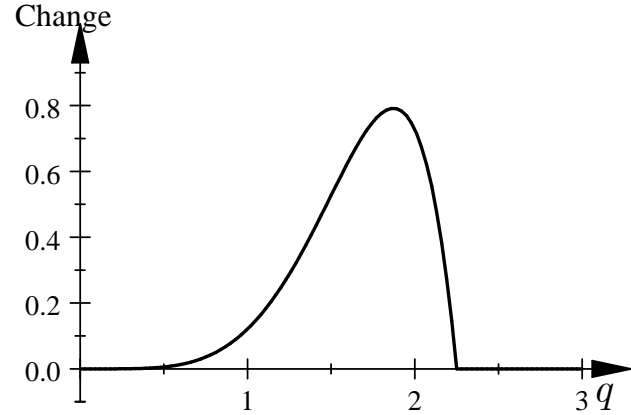

Figure 8: Absolute change in social welfare from removal of copyright; scenario 2

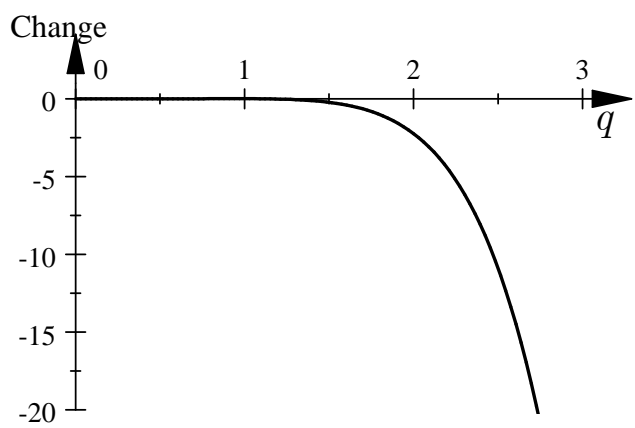

Figure 9: Absolute change in social welfare from removal of copyright; scenario 3

Finally, we comment on the last row of Table 2. The relative change in the share of welfare that goes to academics is decreasing in quality in scenario 2 and increasing in quality in scenario 3 . If copyright is removed while we are in scenario 2, total welfare is likely to go up. However, the share of welfare that accrues to academics drops. If we are in scenario 3 , the share of academic welfare in total welfare rises when copyright is removed, but it is more likely that total welfare drops. We are also able to perform a welfare analysis for readers and authors separately. It turns out that the removal of copyright in either scenario leads to less author welfare and more reader welfare, and the gain in reader welfare outweighs the loss in author welfare. ${ }^{25}$

\section{A consideration of capacity constraints}

Above, we have noted that in order to consider some degree of competition in our model, it would be relevant to impose capacity constraints on both sides of the market. In this way, the journals market can be considered as a market of monopolistic competition. A full consideration of capacity

\footnotetext{
${ }^{24}$ Again this positive part cannot be discerned in the graph, unless we change the vertical scale by a factor of about $\frac{1}{100}$.

${ }^{25}$ If we were to consider academics at different universities, and since the authorship at some universities is significantly higher than at others (high-ranked universities versus low-ranked ones on a scale of publications), then we might want to calculate reader and author welfare separately. At universities with low publication outputs, the academics are mainly readers. These universities would apparently gain significantly from removal of copyright. The same may not be true in universities with a high number of publications. See Mueller-Langer and Watt (2010) for further details.
} 
constraints in the simulations we have conducted would require a large number of new scenarios to consider. We leave this more detailed analysis for future research. However, it is worthwhile to mention how things would play out.

Under a capacity constraint, the journal is bounded by a certain maximum number of both readers and authors. The number of readers and authors are both increasing functions of quality in all of the model configurations that we have used. Thus, although the journal's profit is also increasing in quality, the journal would not be able to set quality arbitrarily high, as at some point it would run out of either readers or authors. In this way, the capacity constraints would determine the final quality achieved in the model.

The introduction of capacity constraints would have important effects when the removal of copyright is considered. Unless the removal of copyright itself is able to alter the binding constraint (which would seem not to be logical), the capacity constraints have the potential to intervene in the welfare analysis of the previous section.

Consider, for example, scenario 1. When copyright is removed, social welfare increases by around $15 \%$ regardless of the level of quality. However, in that scenario, the removal of copyright will also increase the number of readers and authors at each level of quality. This in turn implies that the capacity constraint must now bind at a lower level of quality. Hence, in the end the final quality that is actually achieved decreases. Following the argumentation of McCabe and Snyder (2005, $2007 \& 2013 \mathrm{a})$, author demand is ceteris paribus less likely to be sufficiently inelastic in this case to support a high author price, which is necessary for open access to be a sustainable long-run equilibrium and welfare enhancing. Finally, since social welfare is an increasing function of quality, there is an off-setting effect on social welfare that may or may not counterbalance the $15 \%$ gain that is initially found by removing copyright.

Scenario 2 works in a similar way to scenario 1 regarding this capacity constraint effect. Removal of copyright will initially increase social welfare at almost all levels of quality, but it will also increase the number of both readers and authors at each quality level. Thus the capacity constraint will bind at a lower level of quality, and so final quality achieved will go down. ${ }^{26}$ The social welfare gains are, at least partially, off-set by the welfare loss of a lower quality level. On the other hand, in scenario 3, the opposite occurs. In the first instance, removal of copyright is likely to increase social welfare at each level of quality, but (at least for high enough levels of quality), the number of readers and authors are decreased at each level of quality when copyright is removed (again, for high enough levels of quality). ${ }^{27}$ The capacity constraint then would bind at a higher level of quality than before, implying a welfare gain that (at least partially) off-sets the losses from removal of copyright. In this case, author demand is ceteris paribus more likely to be sufficiently inelastic to support a high author price, which is necessary for open access to be a sustainable long-run

\footnotetext{
${ }^{26}$ Again, this is assuming that the optimal reader price with copyright was not set at 0 . If open access were optimal under copyright, then of course no effect at all happens when copyright is removed.

${ }^{27}$ This only happens in scenario 3 when quality is above a certain threshold. However, the threshold is at a relatively low level of quality, and below this threshold although the numbers of academics served actually increase, the change is rather infinitesimal.
} 
equilibrium and welfare enhancing.

It is impossible to know which of the two effects (the direct welfare effect at each level of quality from removal of copyright, or the indirect welfare effect of the change in quality due to the capacity constraints) is larger. Studying this effect would constitute an interesting extension to the present paper.

\section{Conclusions}

All of our conclusions are based upon numerical simulation and particular functional forms, and thus should be read with due care. The driving mechanism in our model is the effect of an increased level of quality upon the number of readers and authors, that is, the vertical intercept of our demand curves. Different values for this vertical intercept would change our results quantitively, but not qualitatively. We also remind the reader that the results only apply to a local range of quality levels, i.e. the range for which quality increases lead to an increase in the number of authors that are accepted for publication.

Our conclusions are the following. First, it is the nature of the non-linear externalities between authors and readers that determines whether open access is a feature of low or high-quality journals. Indeed, we find that under appropriate profit maximisation on both sides of the journal market, there exist configurations under which it is the higher-quality journals that will have the openaccess format. This is the case in our scenario 2, where the production of readers (taking authors as an input) has diminishing returns while the production of authors (taking readers as an input) is linear. In contrast, open access is never a feature of an optimally priced journal in scenario 1, where both production functions are affected by diminishing returns.

Second, with regard to the hypothesised removal of copyright for academic works, as suggested by Shavell (2010), we find that the removal of copyright will have a different effect depending on the configuration of the market. We find scenarios in which the removal of copyright will have hardly any effect on profits, but will increase social welfare for almost all quality measures (scenario 2). In contrast, the removal of copyright will have a serious negative effect on the profits of high-quality journals such that social welfare decreases in scenario 3. Thus, we cannot unambiguously support the removal of copyright. On the other hand, we cannot unambiguously support its continued

retention. In our scenario 1, we find that the removal of copyright is unambiguously social welfare improving, but it will also have a serious negative effect on journal profits. If the real state of the world is similar to that of scenario 1, then the removal of copyright is likely to be a beneficial social policy, but it may have to be accompanied by an alternative (author-pays) business model for publication of scientific work.

This paper suggests several paths for future research. First, it would be interesting to investigate empirically which, if any, of our three scenarios is most likely to be real-world relevant. Scenarios 1 and 2 provide support for the removal of copyright, while scenario 3 does not. The critical issue is where the diminishing returns lie; is it the production of readers with authors as an input that suffers 
diminishing returns, or rather the production of authors with readers as an input? As indicated in the discussion of the three scenarios, we can think of logical reasons to support either argument. A comprehensive empirical examination should investigate this issue. Second, our model has been calibrated with a single parameter for the effect of increased quality upon the demand for journal space by both authors and readers. While considering different values of this parameter will not alter our model in a significant manner, it would be of interest to consider that the effect is different for authors than for readers. However, it is again very hard to think of convincing reasons why an increase in journal quality will attract new readers in a notably different way to how it attracts new authors. Third, the model generates specific formulas for the number of readers and the number of authors for each quality level. The ratio between these two gives us the number of readers per published paper, something that we may associate with the "impact" of the journal. Further, the impact factor that is habitually used (e.g. by ISI), which is citations per paper published, can be seen as nothing more than readers per paper times the probability that any given reader will end up citing the paper she reads in a follow-up paper. It would be of great interest to identify an appropriate function for the probability of citing (as a function of the quality of the journal article read), so that our model may then be applied directly to an analysis of the validity of the ISI impact factor as an indicator of journal quality. Fourth, it would be interesting to make the number of authors a non-monotone function of quality in order to endogenously derive a single optimal level of quality. Fifth, the journal that we have modelled is an online-only product with zero marginal costs. We would be interested in a version of this model being applied to journals with both hard-print and online formats, and above all, a journal with a hybrid open-access policy (a policy in which the author can decide, and pay a corresponding fee to the journal, in order to have the article priced at zero to readers). Finally, for now we have preferred to look at a monopolistic journal (or at most, a monpolistically competitive journal) in order to focus on the issue of open access and copyright. Our model has paid scant attention to competition over journals and the precise manner in which quality is chosen (i.e. the referee process). Accounting for either or both of these features would improve the model, although we hypothesise at a significant increase in complexity. 


\section{References}

[1] Armstrong, M. (2006), "Competition in Two-sided Markets", RAND Journal of Economics, $37(3), 668-91$.

[2] Bergstrom, T. (2001), "Free Labor for Costly Journals?", Journal of Economic Perspectives, $15(4), 183-98$.

[3] Dewatripont, M., et al. (2006). Study on the Economic and Technical Evolution of the Scientific Publication Markets in Europe. Brussels: European Commission Directorate General for Research.

[4] Ellison, G. (2002), "The Slowdown of the Economics Publishing Process", Journal of Political Economy, 110(5), 947-93.

[5] Filistrucchi, L., D. Geradin and E. van Damme (2013), "Identifying Two-sided Markets", World Competition, 36(1), 33-59.

[6] Gans, J., Editor, (2000), "Publishing Economics: Analyses of the Academic Journal Market in Economics", Cheltenham, UK: Edward Elgar.

[7] Hamermesh, D. S. (1992), "The Young Economist's Guide to Professional Etiquette", Journal of Economic Perspectives, 6(1), 169-179.

[8] Heintzelman, M. and D. Nocetti (2009), "Where Should we Submit our Manuscript? An Analysis of Journal Submission Strategies," B.E. Journal of Economic Analysis and Policy, 9(1), Advances, Article 39.

[9] Hilty, R.M. (2005), "Five Lessons about Copyright in the Information Society: Reaction of the Scientific Community to Over-Protection and what Policy Makers Should Learn," Journal of the Copyright Society of the USA, 53(1-2), 103-38.

[10] Jeon D.S. and D. Menicucci (2006), "Bundling Electronic Journals and Competition among Publishers", Journal of the European Economic Association, 4(5), 1038-83.

[11] Jeon, D.S. and J.C. Rochet (2010), "The Pricing of Academic Journals: A Two-sided Market Perspective", American Economic Journal: Microeconomics, 2, 222-55.

[12] Kóczy, Á.L. and A. Nichifor (2013), "The Intellectual Influence of Economic Journals: Quality and Quantity", Economic Theory, 52(3), 863-84.

[13] McCabe, M.J. (2004), "Information Goods and Endogenous Pricing Strategies: The Case of Academic Journals", Economics Bulletin, 12(10), 1-11.

[14] McCabe, M.J. and C.M. Snyder (2005), "Open Access and Academic Journal Quality", American Economic Review, Papers and Proceedings, 95(2), 453-8. 
[15] McCabe, M.J. and C.M. Snyder (2007), "Academic Journal Prices in a Digital Age: A TwoSided Market Model", The B.E. Journal of Economic Analysis and Policy, 7(1), Contributions, Article 2.

[16] McCabe, M.J. and C.M. Snyder (2013a), "The Rich Get Richer and the Poor Get Poorer: The Effect of Open Access on Cites to Science Journals Across the Quality Spectrum", Working Paper (May 2013), available at: http://mccabe.people.si.umich.edu/.

[17] McCabe, M.J. and C.M. Snyder (2013b), "Does Online Availability Increase Citations? Theory and Evidence from a Panel of Economics and Business Journals?", Working Paper (March 2013), Revise and Resubmit, Review of Economics and Statistics, available at: http://mccabe.people.si.umich.edu/.

[18] McCabe, M.J., C.M. Snyder and A. Fagin (2013), "Open Access versus Traditional Journal Pricing: Using a Simple "Platform Market" Model to Understand Which Will Win (and Which Should)", Journal of Academic Librarianship, 39, 11-19.

[19] Mueller-Langer, F. and R. Watt (2010), "Copyright and Open Access for Academic Works", Review of Economic Research on Copyright Issues, 7(1), 45-65.

[20] Oster, S. (1980), "The Optimal Order for Submitting Manuscripts", American Economic Review, 70(3), 444-448.

[21] Parker, G.G. and M.W. Van Alstyne (2005), "Two-sided Network Effects: A Theory of Information Product Design", Management Science, 51(10), 1494-1504.

[22] Rochet, J.C. and J. Tirole (2002), "Cooperation among Competitors: Some Economics of Payment Card Associations", RAND Journal of Economics, 33(4), 549-70.

[23] Rochet, J.C. and J. Tirole (2006), "Two-sided Markets: A Progress Report", RAND Journal of Economics, 35(3), 645-67.

[24] Rysman, M. (2009), "The Economics of Two-sided Markets", Journal of Economic Perspectives, 23(3), 125-43.

[25] Shavell, S. (2010), "Should Copyright of Academic Works be Abolished?", Journal of Legal Analysis, 2(1), 301-58.

[26] Suber, P. (2012), "Open Access", Cambridge, MA: MIT Press Essential Knowledge.

[27] Towse, R., C. Handke and P. Stepan (2008), “'The Economics of Copyright Law: A Stocktake of the Literature, Review of Economic Research on Copyright Issues, 5(1), 1-22. 


\section{Appendix A: Optimal prices in scenario 2 under copyright protection}

The two simultaneous equations $n_{r}=\sqrt{n_{a}}\left(\alpha q-p_{r}\right)$ and $n_{a}=n_{r}\left(\alpha q-p_{a}\right)$ can be written as

$$
\begin{aligned}
& n_{r}=\sqrt{n_{a}} \beta_{r}, \\
& n_{a}=n_{r} \beta_{a},
\end{aligned}
$$

where $\beta_{i} \equiv \alpha q-p_{i}$ for $i=r, a$. Both $n_{r}$ and $n_{a}$ are constrained to be positive. We are restricted to parameter values such that $\beta_{i}>0$ for $i=r, a$. That is, we can only consider prices that satisfy $p_{i}<\alpha q$ for $i=r, a$. It is easy to show that the solution to the two equations (5) and (6), outside the trivial solution at $(0,0)$, lies at $n_{r}=\beta_{r}^{2} \beta_{a} ; n_{a}=\beta_{r}^{2} \beta_{a}^{2}$. The profits of the journal are given by $\pi=p_{r} n_{r}+p_{a} n_{a}=p_{r} \beta_{r}^{2} \beta_{a}+p_{a} \beta_{r}^{2} \beta_{a}^{2}$. From the definitions of the two $\beta_{i}$ functions, we can see that profit is now a third-order function of each price.

First, consider the optimal reader price. The two derivatives of the profit function with respect to $p_{r}$ are:

$$
\begin{aligned}
& \frac{\partial \pi}{\partial p_{r}}=\beta_{r}^{2} \beta_{a}-p_{r} 2 \beta_{r} \beta_{a}-p_{a} 2 \beta_{r} \beta_{a}^{2} \\
& \frac{\partial^{2} \pi}{\partial p_{r}^{2}}=-4 \beta_{r} \beta_{a}+p_{r} 2 \beta_{a}+p_{a} 2 \beta_{a}^{2}
\end{aligned}
$$

The first-order condition for a maximum is $\frac{\partial \pi}{\partial p_{r}^{*}}=0 \Rightarrow \beta_{r}^{* 2} \beta_{a}-p_{r}^{*} 2 \beta_{r}^{*} \beta_{a}-p_{a} 2 \beta_{r}^{*} \beta_{a}^{2}=0$, where $\beta_{r}^{*}=\alpha q-p_{r}^{*}$. Extracting the common factor, we have $\beta_{r}^{*} \beta_{a}\left(\beta_{r}^{*}-2 p_{r}^{*}-2 p_{a} \beta_{a}\right)=0$. Since $\beta_{r}^{*} \beta_{a}>0$, we have

$$
\beta_{r}^{*}-2 p_{r}^{*}-2 p_{a} \beta_{a}=0
$$

Substituting for $\beta_{r}^{*}$, this reads $\alpha q-p_{r}-2 p_{r}-2 p_{a}\left(\alpha q-p_{a}\right)=0$. The final solution is given by

$$
p_{r}^{*}=\frac{\alpha q-2 p_{a}\left(\alpha q-p_{a}\right)}{3} .
$$

This solution is unique on the range $p_{r}<\alpha q$. Since our solution (8) is unique, in order to ensure that it is a maximum, we need to show that the second-order condition holds at that solution:

$$
\frac{\partial^{2} \pi}{\partial p_{r}^{2}}=-4 \beta_{r}^{*} \beta_{a}+2 p_{r}^{*} \beta_{a}+2 p_{a} \beta_{a}^{2}<0 \Rightarrow-4 \beta_{r}^{*}+2 p_{r}^{*}+2 p_{a} \beta_{a}<0 .
$$

Equation (7) is $2 p_{a} \beta_{a}=\beta_{r}^{*}-2 p_{r}^{*}$. Substituting this into our second-order condition we get $-4 \beta_{r}^{*}+2 p_{r}^{*}+\beta_{r}^{*}-2 p_{r}^{*}<0 \Rightarrow-3 \beta_{r}^{*}<0$, which holds for any $p_{r}^{*}<\alpha q$. Thus, (8) is indeed a maximum.

Second, consider the optimal author price. The first two derivatives of the profit function with 
respect to $p_{a}$ are:

$$
\begin{aligned}
\frac{\partial \pi}{\partial p_{a}} & =-p_{r} \beta_{r}^{2}+\beta_{r}^{2} \beta_{a}^{2}-2 p_{a} \beta_{r}^{2} \beta_{a} \\
\frac{\partial^{2} \pi}{\partial p_{a}^{2}} & =-4 \beta_{r}^{2} \beta_{a}+2 p_{a} \beta_{r}^{2} .
\end{aligned}
$$

The first-order condition is

$$
-p_{r} \beta_{r}^{2}+\beta_{r}^{2} \beta_{a}^{2 *}-2 p_{a}^{*} \beta_{r}^{2} \beta_{a}^{*}=0 \Rightarrow-p_{r}+\beta_{a}^{2 *}-2 p_{a}^{*} \beta_{a}^{*}=0 .
$$

The second order condition is $-4 \beta_{a}^{*}+2 p_{a}^{*}<0$, which, upon substituting for $\beta_{a}^{*}$ reduces to

$$
p_{a}^{*}<\frac{2 \alpha q}{3} .
$$

Now, note that $(9)$ is just $-p_{r}+\left(\alpha q-p_{a}^{*}\right)^{2}-2 p_{a}^{*}\left(\alpha q-p_{a}^{*}\right)=0$ or $3 p_{a}^{* 2}-4 \alpha q p_{a}^{*}-p_{r}+$ $(\alpha q)^{2}=0$. Using the quadratic formula, we know that the two roots of this equation satisfy $\frac{4 \alpha q \pm \sqrt{16 \alpha^{2} q^{2}-12\left(\alpha^{2} q^{2}-p_{r}\right)}}{6}$. Simplifying, we get $\frac{2 \alpha q \pm \sqrt{\alpha^{2} q^{2}+3 p_{r}}}{3}=\frac{2 \alpha q}{3} \pm \frac{\sqrt{\alpha^{2} q^{2}+3 p_{r}}}{3}$. Using the secondorder condition we see that the higher of these two roots is a minimum whereas the lower is the maximum. Thus, the optimal author price is given by

$$
p_{a}^{*}=\frac{2 \alpha q-\sqrt{\alpha^{2} q^{2}+3 p_{r}}}{3} .
$$

In order to find the exact optimal prices for readers and authors as functions of the journal quality $q$, we simultaneously solve the two relevant first-order equations:

$$
p_{a}^{*}=\frac{2 \alpha q-\sqrt{\alpha^{2} q^{2}+3\left(\frac{\alpha q-2 p_{a}^{*}\left(\alpha q-p_{a}^{*}\right)}{3}\right)}}{3}=\frac{2 \alpha q-\sqrt{\alpha^{2} q^{2}+\alpha q-2 p_{a}^{*}\left(\alpha q-p_{a}^{*}\right)}}{3} .
$$

Simple steps then give

$$
\begin{aligned}
2 \alpha q-3 p_{a}^{*} & =\sqrt{\alpha^{2} q^{2}+\alpha q-2 p_{a}^{*}\left(\alpha q-p_{a}^{*}\right)} \\
& \Rightarrow 4 \alpha^{2} q^{2}-12 \alpha q p_{a}^{*}+9 p_{a}^{* 2}=\alpha^{2} q^{2}+\alpha q-2 p_{a}^{*}\left(\alpha q-p_{a}^{*}\right) .
\end{aligned}
$$

We get the following second-order equation:

$$
7 p_{a}^{* 2}-10 \alpha q p_{a}^{*}+3 \alpha^{2} q^{2}-\alpha q=0
$$

Applying the quadratic formula, we get

$$
p_{a}^{*}=\frac{10 \alpha q \pm \sqrt{100 \alpha^{2} q^{2}-28\left(3 \alpha^{2} q^{2}-\alpha q\right)}}{14}=\frac{5 \alpha q \pm \sqrt{\left.4 \alpha^{2} q^{2}+7 \alpha q\right)}}{7} .
$$


The upper root of this is greater $\operatorname{than}^{28} \alpha q$. So the unique value of $p_{a}^{*}$ is

$$
p_{a}^{*}=\frac{5 \alpha q-\sqrt{\left.4 \alpha^{2} q^{2}+7 \alpha q\right)}}{7} .
$$

Finally then, we need to substitute this back in to the equation for the optimal reader price $(8):{ }^{29}$

$$
\begin{aligned}
p_{r}^{*} & =\frac{\alpha q-2\left(\frac{5 \alpha q-\sqrt{\left.4 \alpha^{2} q^{2}+7 \alpha q\right)}}{7}\right)\left(\alpha q-\left(\frac{5 \alpha q-\sqrt{\left.4 \alpha^{2} q^{2}+7 \alpha q\right)}}{7}\right)\right)}{3} \\
& =\frac{21 \alpha q-4 \alpha^{2} q^{2}-2 \alpha q \sqrt{q\left(4 \alpha^{2} q+7 \alpha\right)}}{49} .
\end{aligned}
$$

\section{Appendix B: Optimal author prices when copyright is removed}

Scenario 1 When copyright is removed, and the reader price is constrained to be equal to 0 , the profit of the journal is given by $\pi=p_{a} n_{a}=p_{a}\left(\beta_{r}^{2} \beta_{a}^{4}\right)^{\frac{1}{3}}$. We have $\beta_{r}=\alpha q$, so the profit function can be written as $\pi=p_{a}\left((\alpha q)^{2} \beta_{a}^{4}\right)^{\frac{1}{3}}=(\alpha q)^{\frac{2}{3}} p_{a} \beta_{a}^{\frac{4}{3}}$. The first-order condition ${ }^{30}$ for an optimal choice of $p_{a}$ is $(\alpha q)^{\frac{2}{3}}\left(\beta_{a}^{* \frac{4}{3}}-\frac{4}{3} p_{a}^{*} \beta_{a}^{* \frac{1}{3}}\right)=0 \Rightarrow \beta_{a}^{*}=\frac{4}{3} p_{a}^{*}$, which, since $\beta_{a}^{*}=\left(\alpha q-p_{a}^{*}\right)$, is the same as $p_{a}^{*}=\frac{3 \alpha q}{7}$. Recall that under copyright, the optimal author price was $\frac{\alpha q}{3}$, thus aside from reducing the reader price to 0 , the removal of copyright serves to increase the optimal author price by $\frac{3 \alpha q}{7}-\frac{\alpha q}{3}=\frac{2 \alpha q}{21}$.

Scenario 2 There is no need to re-do the optimisation under the restriction that $p_{r}=0$. We only need to use that value of reader price in the equation (11) in Appendix A. Substituting in $p_{r}=0$, and simplifying, we see that the optimal price without copyright is given by $p_{a}^{*}=\frac{\alpha q}{3}$. Again, the optimal author price increases with the removal of copyright. In contrast to the case of copyright protection, now the optimal author price is strictly positive and linear for all levels of quality.

Scenario 3 The relevant equation from Appendix A (with the subscripts switched to capture the modelling change) is $p_{a}^{*}=\frac{\alpha q-2 p_{r}\left(\alpha q-p_{r}\right)}{3}$. Clearly, setting $p_{r}=0$ gives us exactly the same author price as in scenario 2 , namely $p_{a}^{*}=\frac{\alpha q}{3}$.

\footnotetext{
${ }^{28}$ The upper root is $\frac{5 \alpha q}{7}+\frac{1}{7} \sqrt{4 \alpha^{2} q^{2}+7 \alpha q}>\frac{5 \alpha q}{7}+\frac{1}{7} \sqrt{4 \alpha^{2} q^{2}}=\frac{5 \alpha q}{7}+\frac{2 \alpha q}{7}=\alpha q$.

${ }^{29}$ The simplification for this was carried out using the package Mathematica.

${ }^{30}$ The second-order condition is $-\frac{8}{3} \beta_{a}^{* \frac{1}{3}}+\frac{4}{9} p_{a}^{*} \beta_{a}^{*-\frac{2}{3}}<0$. This is satisfied if $p_{a}^{*}<6 \beta_{a}^{*}$. Using the definition of $\beta_{a}^{*}$, the second order condition can be written as $p_{a}^{*}<\frac{6 \alpha q}{7}$. The solution to the first-order condition satisfies this, and so we can be assured that $p_{a}^{*}$ is indeed a maximum.
} 